\title{
Hepatic blood flow by perfusion computed tomography as an imaging biomarker for patients with gastric cancer
}

\author{
KIYOHIKO SHUTO $^{1}$, MIKITO MORI ${ }^{1}$, CHIHIRO KOSUGI $^{1}$, KAZUO NARUSHIMA ${ }^{1}$, SATOKO NAKABAYASHI ${ }^{1}$, \\ TAKESHI FUJISIRO ${ }^{2}$, ASAMI SATO ${ }^{2}$, KOICHI HAYANO ${ }^{2}$, HIROAKI SHIMIZU $^{2}$ and KEIJI KODA ${ }^{1}$ \\ ${ }^{1}$ Department of Surgery, Teikyo University Chiba Medical Center, Ichihara, Chiba 299-0111; \\ ${ }^{2}$ Department of Frontier Surgery, Chiba University Graduate School of Medicine, Chiba 260-8670, Japan
}

Received August 15, 2018; Accepted January 17, 2019

DOI: $10.3892 / \mathrm{ol} .2019 .9969$

\begin{abstract}
Perfusion computed tomography (PCT) is a less invasive imaging modality that provides information about tissue hemodynamics at the capillary level. The present study aimed to investigate the correlation between hepatic perfusion and gastric cancer progression. A total of 136 patients with gastric adenocarcinoma were evaluated in the present study. Prior to initial treatment, liver PCT was performed across the hepatic hilar plane and the hepatic blood flow (HBF) was measured using the dual-input deconvolution method. HBF was compared with clinicopathological factors, patient prognosis and circulating serum proangiogenic cytokines. The median $\mathrm{HBF}$ was $217 \mathrm{ml} / \mathrm{min} / 100 \mathrm{~g}$ tissue. Patients with high HBF had larger tumors (43 mm vs. 71, P<0.001) and more advanced tumor-node stages ( $\mathrm{P}<0.001$ for both). When both patient groups of operable and inoperable were compared by their respective median $\mathrm{HBF}$ values, each high-HBF group had a significantly worse prognosis $(\mathrm{P}=0.002$ and $\mathrm{P}=0.024)$, notably in the inoperable group, with $<1$-year survival. In 17 postoperative recurrent patients, the high-HBF at recurrence group also had a significantly worse postrecurrent prognosis $(\mathrm{P}=0.019)$. HBF was an independent prognostic factor (hazard ratio, 2.019; $\mathrm{P}=0.048)$ and was strongly associated with serum vascular endothelial growth factor level $(\mathrm{R}=0.607, \mathrm{P}<0.001)$. $\mathrm{HBF}$ was significantly correlated with gastric cancer progression, and is an easily measured imaging biomarker reflecting patient survival.
\end{abstract}

\section{Introduction}

Locally advanced gastric cancer is the third leading cause of cancer-related death worldwide (1). In the early stages, gastric

Correspondence to: Dr Kiyohiko Shuto, Department of Surgery, Teikyo University Chiba Medical Center, 3426-3 Anesaki, Ichihara, Chiba 299-0111, Japan

E-mail: kshuto@med.teikyo-u.ac.jp

Key words: gastric cancer, biomarker, liver, perfusion, computed tomography, blood flow cancer can often be cured by complete surgical resection with lymphadenectomy, whereas patients with advanced cancer have a poor prognosis. Although the Adjuvant Chemotherapy Trial of S-1 for Gastric Cancer, a randomized phase III trial in patients with stage II/III gastric cancer revealed a prolonged 5 -year survival rate of $50-84 \%$ (2), the prognosis of patients with metastatic and recurrent cancer remains poor with a median overall survival of approximately 1 year $(3,4)$. Variety different metastatic patterns, such as hematogenous, lymphogenic, and disseminated metastasis, prevent proper treatment and result in a poor prognosis. Identifying a more effective and easy-to-use clinical marker may lead to more effective multidisciplinary treatments and more optimal postoperative surveillance.

Cancer progression produces many systemic biological reactions including metabolic disorders, neuroendocrine responses, and cytokine expression (5-7). Angiogenesis plays a fundamental role in tumor growth, malignant transformation, and invasion of tumor cells $(8,9)$, and overexpression of proangiogenic cytokines, such as vascular endothelial growth factor (VEGF), epidermal growth factor receptor (EGFR), platelet-derived endothelial cell growth factor, and hepatocyte growth factor (HGF), is correlated with increased cancer infiltration and poor prognosis in patients with gastric cancer (10-14). VEGF is a key regulator of tumor-associated angiogenesis. Recent reports have demonstrated that the circulating serum level of VEGF is correlated with recurrence and survival in patients with gastric cancer and is thus, a useful biomarker for postoperative follow-up and monitoring patients' responses to chemotherapy (15-18). Systemic changes in tissue circulation may be expected secondary to circulating proangiogenic cytokines; however, the relationship between tissue hemodynamic changes and cancer progression has not been reported.

Perfusion computed tomography (PCT) is a less invasive imaging modality that provides information about tissue hemodynamics at the capillary level in quantitative procedures. Since PCT was first performed in a patient with stroke (19), it has been applied clinically in a variety of liver conditions (20-22), and recent reports have discussed its clinical utility for cancer characterization, predicting the response to chemoradiotherapy, assessing the response to these therapies, and predicting patient outcomes (23-25). 
In this study, we hypothesized that cancer-associated systemic changes secondary to biological reactions and circulating cytokines might affect and alter tissue circulation. Therefore, we investigated hepatic blood flow (HBF) as a surrogate marker because the liver, as an abundant blood-supplied organ, may amplify changes in systemic tissue perfusion, and may be an appropriate and easy target organ to assess by CT.

\section{Materials and methods}

Patientpopulation. This study was approved by the institutional review board (IRB) of the Medical Ethical Committee of Teikyo University (Ichihara, Japan), and written informed consent was obtained from all patients (IRB number, 12-179; UMIN000019970). We enrolled 166 consecutive patients with histologically-proven gastric adenocarcinoma who were treated at Teikyo University Chiba Medical Center, Ichihara, Japan from March 2013 to December 2017. The eligibility criteria were as follows: (i) age $\geq 20$ years; (ii) no liver dysfunction, cirrhosis, or jaundice (serum alanine aminotransferase level $<120 \mathrm{IU} / \mathrm{l}$, serum total bilirubin level $<2.0 \mathrm{mg} / \mathrm{dl}$, and blood platelet count $>150,000 / \mu 1$ ); (iii) adequate renal function (serum creatinine level $<2.0 \mathrm{mg} / \mathrm{dl}$ ); (iv) absence of multiple liver metastases ( $\leq 3 \mathrm{~cm}$ in diameter and $\leq 3$ lesions); (v) no inflammation (C-reactive protein level $<3.0 \mathrm{mg} / \mathrm{dl}$ and white blood cell count $<12,000 / \mu l$ ); and (vi) no other history of active malignant neoplasms. We excluded 30 patients whose acquired images were not suitable for analysis because of an insufficient concentration of the contrast agent or excessive respiratory movements $(n=18)$ or who refused medical intervention $(\mathrm{n}=12)$. Finally, we included 136 patients; $15(11 \%)$ patients had synchronous liver metastasis, and the median follow-up duration was 685 days (range, 17-1923 days) (Table I).

Cancer staging and treatment. The location and size of the tumors were clinically determined by gastrointestinal endoscopy and biopsy specimens, contrast-enhanced CT, magnetic resonance imaging, and barium contrast radiography. The tumor-node-metastasis (TNM) classification of the Union for International Cancer Control (26) was used for evaluation. In total, 103 patients underwent surgical resection; the remaining 33 had inoperable disease. In the operable group, 24 received two or three cycles of neoadjuvant chemotherapy using CS (S-1 with cisplatin) or SOX (S-1 with oxaliplatin). Gastrectomy with D0 or D1 lymph node dissection, including palliative resection, was performed for clinical T1a, N0 cancer and stage IV disease, otherwise we performed gastrectomy with $\mathrm{D} 1+$ or D2 dissection. The extent of lymph node dissection was determined according to the Japanese Classification of Gastric Carcinoma (27). Patients with pathological stage II/III disease received postoperative adjuvant chemotherapy with S-1 for 1 year. In the inoperable group, CS or SOX were administered as first-line chemotherapy, and ramucirumab and paclitaxel as second-line therapies, if possible (Table I).

Liver PCT imaging protocol. Liver PCT was performed with a 64-row multidetector CT scanner (LightSpeed VCT; GE Medical Systems, Milwaukee, WI, USA). After checking patients' vital signs, we administered supplemental oxygen at $3 \mathrm{l} / \mathrm{min}$ for $10 \mathrm{~min}$ through a venturi mask in the supine position on the scanner table. A non-enhanced CT scan was obtained for initial liver localization. Eight sequential 5 -mm-slice scans with a total length of 4-cm thickness of the liver, including the hepatic hilum, were selected. Contrast material (370 mg iodine/ml, Iopamiron 370; Bayer Healthcare, Leverkusen, Germany) was administered to a total dose of $0.8 \mathrm{ml} / \mathrm{kg}$ at a rate of $5 \mathrm{ml} / \mathrm{s}$ with a $20-\mathrm{ml}$ saline chaser using a power injector (Dual Shot GX; Nemoto Kyorindo, Tokyo, Japan). A dynamic study of the selected slices was then performed during a breath hold at a static table position. Images were obtained with the following parameters: $10-\mathrm{sec}$ start delay, $40-\mathrm{mm}$ beam collimation, $120-\mathrm{kV}$ tube voltage, 60-mA tube current, 1-sec gantry rotation time, and 1-sec inter-scan delay. PCT was performed before initial treatment in all patients and at 6-month intervals postoperatively in patients who underwent gastrectomy.

Imaging interpretation. Image data were transferred to an image-processing workstation (Advantage Workstation ver. 4.4; GE Medical Systems) and analyzed by the commercially-based CT perfusion software of a noncompartment model with a dual-input deconvolution method (CT Perfusion ver. 4; GE Medical Systems). Analyses were based on the following principles: Contrast material injected as a rapid bolus fills the entire capillary network, arrives at equilibrium, and then washes out from the tissue network. Because iodinated contrast material can be considered a purely intravascular tracer on dynamic $\mathrm{CT}$, its concentration within tissue can be obtained by the arterial and portal input; change in contrast material volume is shown as the impulse retention function (28). Input perfusion data were determined by placing a region of interest in the abdominal aorta (median area, $121 \mathrm{~mm}^{2}$; range, 22-328 $\mathrm{mm}^{2}$ ) and in the portal vein trunk (median area, $33 \mathrm{~mm}^{2}$; range, $10-99 \mathrm{~mm}^{2}$ ). Time-density curves of the abdominal aorta and the portal vein were automatically generated for blood input, and the data were processed into a functional map representing $\mathrm{HBF}(\mathrm{ml} / \mathrm{min} / 100 \mathrm{~g}$ tissue).

HBF was calculated in the workstation, and a functional map was displayed in colors ranging from blue to red with red being the higher range. A freehand region of interest in the liver was traced on enhanced gray-scale raw CT images according to the following rules: A line was drawn around the entire right hepatic lobe, including the medial segment, if possible; a 1-2-cm margin from the hepatic surface was ensured; and intrahepatic lesions, if present, were excluded (e.g., cyst, hemangioma, and metastatic lesions). HBF values in the four central slices, which were selected to include the maximum hepatic plane, were then averaged and used for evaluation (Fig. 1).

Analysis of serum proangiogenic factors. Serum proangiogenic cytokines were investigated in the last 40 patients who participated in an additional research project under IRB approval and with written informed consent (IRB number, 15-007; UMIN000019973). Peripheral blood samples were collected and centrifuged to separate the serum, which was then stored at $-80^{\circ} \mathrm{C}$. Electrochemiluminescence immunoassays were used for detection. Serum samples were tested in 
Table I. Characteristics of patients with gastric cancer.

\begin{tabular}{|c|c|}
\hline Total characteristics & Total $(n=136)$ \\
\hline Age (years) & $72(40-90)$ \\
\hline \multicolumn{2}{|l|}{ Sex } \\
\hline Male & 110 \\
\hline Female & 26 \\
\hline \multicolumn{2}{|l|}{ Tumor location } \\
\hline Upper & 32 \\
\hline Middle & 48 \\
\hline Lower & 56 \\
\hline \multicolumn{2}{|l|}{ Histological type } \\
\hline Intestinal & 60 \\
\hline Diffuse & 76 \\
\hline Tumor size (mm) & $55(7-180)$ \\
\hline \multicolumn{2}{|l|}{ Clinical stage } \\
\hline I & 42 \\
\hline II & 20 \\
\hline III & 32 \\
\hline IV & 42 \\
\hline Operable patients & 103 \\
\hline \multicolumn{2}{|l|}{ Neoadjuvant chemotherapy } \\
\hline Yes & 24 \\
\hline No & 79 \\
\hline \multicolumn{2}{|l|}{ Extent of gastrectomy } \\
\hline Distal-proximal gastrectomy & 75 \\
\hline Total gastrectomy & 28 \\
\hline \multicolumn{2}{|l|}{ Pathological stage } \\
\hline $\mathrm{I}$ & 43 \\
\hline II & 21 \\
\hline III & 25 \\
\hline IV & 14 \\
\hline Inoperable patients & 33 \\
\hline \multicolumn{2}{|l|}{ Main cause of inoperability } \\
\hline Dissemination & 15 \\
\hline Distant metastasis & 14 \\
\hline Infiltration into adjacent organs & 4 \\
\hline \multicolumn{2}{|l|}{ Treatment } \\
\hline Chemotherapy only & 23 \\
\hline Chemotherapy + bypass operation & 10 \\
\hline
\end{tabular}

Continuous values are expressed as median (range).

duplicate for concentrations of VEGF, VEGF receptor-2 (VEGFR-2), HGF, and angiopoietin-1 under standard conditions. The following enzyme-linked immunosorbent assays (ELISA) (DuoSet ${ }^{\mathrm{TM}}$ ELISA; R\&D Systems, Minneapolis, MN, USA) were performed to quantify serum levels using standard curves for concentration calculation according to the manufacturer's protocols: DuoSet ELISA Human VEGF (DY293B), DuoSet ELISA Human VEGFR2/KDR (DY357), DuoSet ELISA Human HGF (DY294), and DuoSet ELISA Human Angiopoietin-1 (DY923). ELISA readings were measured at
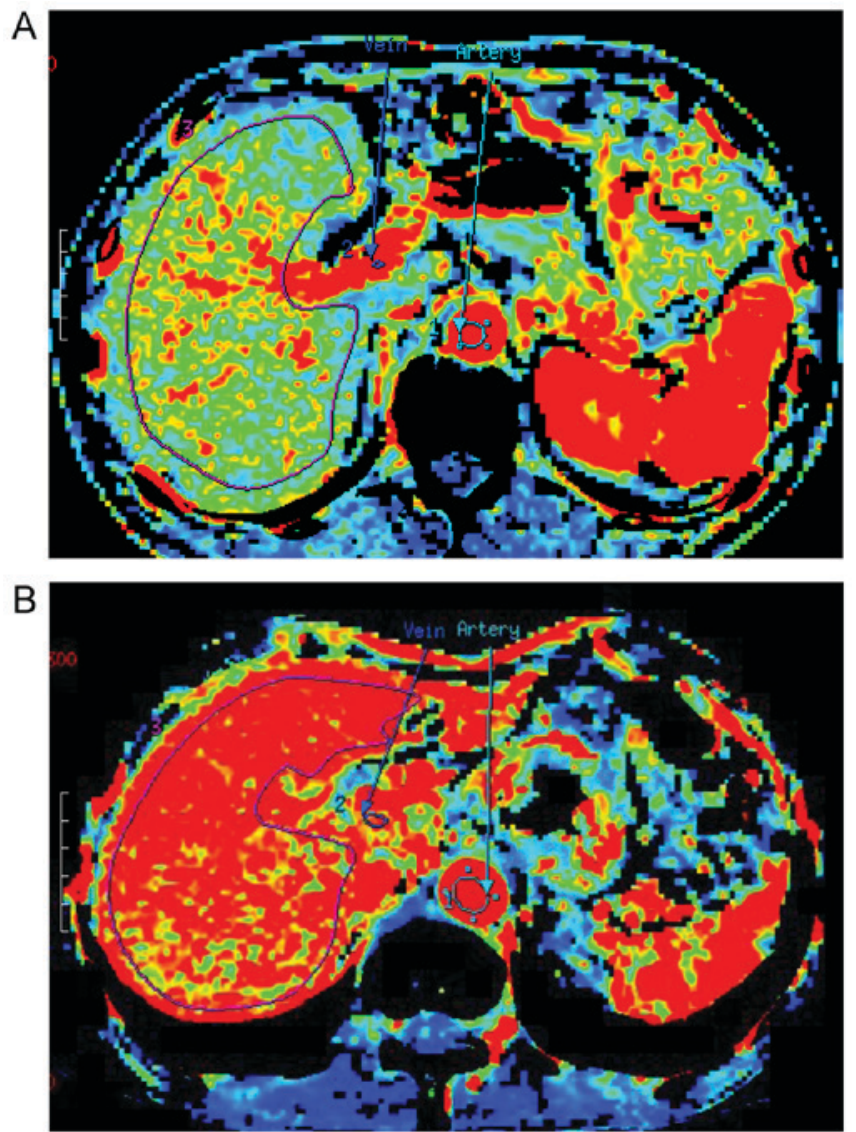

Figure 1. Hepatic blood flow (HBF) maps of liver perfusion CT. (A) A patient with stage I cancer (T1, N0) with preoperative HBF of $158 \mathrm{ml} / \mathrm{min} / 100 \mathrm{~g}$ tissue. (B) A patient with stage IV cancer who underwent laparotomy that revealed unresectable cancer secondary to dissemination, with preoperative $\mathrm{HBF}$ of $310 \mathrm{ml} / \mathrm{min} / 100 \mathrm{~g}$ tissue.

$450 \mathrm{~nm}$ in a microplate reader (ARVO X multilabel reader; PerkinElmer, Waltham, MA, USA).

Statistical analysis. Statistical significance was evaluated by the Mann-Whitney U test for comparison of two continuous parameters and the Chi-square test for categorical variables. One-way analysis of variance was used to analyze the differences among three or more groups performing the Dunnett's test. The correlations between two continuous values were plotted by a linear regression model and evaluated by Pearson's correlation test. A Cox proportional hazard regression model was used to analyze prognostic factors in univariate and multivariate analyses. A multiple logistic regression was calculated to predict $\mathrm{HBF}$ based on significant clinical factors with univariate analyses. Overall survival curves were plotted by the Kaplan-Meier method, and the differences were statistically evaluated by the log-rank test. Two-tailed P-values <0.05 were considered significant. All statistical analyses were performed using SPSS v.22.0 (IBM Corp., Armonk, NY, USA).

\section{Results}

HBF and clinicopathological factors. The median HBF was $217 \mathrm{ml} / \mathrm{min} / 100 \mathrm{~g}$ tissue (range, 128-430 ml/min/100 g tissue). 
Table II. Comparison between hepatic blood flow and clinicopathological factors.

\begin{tabular}{|c|c|c|c|}
\hline \multirow[b]{2}{*}{ Variables } & \multicolumn{2}{|c|}{ Category of HBF } & \multirow[b]{2}{*}{ P-value } \\
\hline & Low $(n=67)$ & High $(n=69)$ & \\
\hline Age (years) & $73(51-89)$ & $69(40-90)$ & 0.230 \\
\hline Gender, $n(\%)$ & & & 0.097 \\
\hline Male & $58(87)$ & $52(75)$ & \\
\hline Female & $9(13)$ & $17(25)$ & \\
\hline BMI $\left(\mathrm{kg} / \mathrm{mm}^{2}\right)$ & $22.7(16.0-34.2)$ & $20.1(12.9-27.4)$ & $<0.001$ \\
\hline $\mathrm{Hb}(\mathrm{g} / \mathrm{dl})$ & $13.9(7.6-17.1)$ & $11.3(7.3-15.2)$ & $<0.001$ \\
\hline AST (IU/l) & $21(12-70)$ & $20(12-85)$ & 0.440 \\
\hline ALT (IU/l) & $18(7-88)$ & $15(5-68)$ & 0.081 \\
\hline Cre $(\mathrm{mg} / \mathrm{dl})$ & $0.81(0.40-1.52)$ & $0.77(0.26-1.40)$ & 0.143 \\
\hline $\mathrm{SBP}(\mathrm{mmHg})$ & $123(93-160)$ & $116(90-158)$ & 0.146 \\
\hline Heart rate $(\mathrm{bpm})$ & $74(48-103)$ & $76(58-111)$ & 0.299 \\
\hline Ejection fraction (\%) & $64.8(40.0-78.4)$ & $63.7(33.2-83.1)$ & 0.442 \\
\hline Valvular heart disease, $n(\%)$ & & & 0.295 \\
\hline Yes & $23(34)$ & $18(26)$ & \\
\hline No & $44(66)$ & $51(74)$ & \\
\hline Tumor location, $n(\%)$ & & & 0.471 \\
\hline Upper & $14(21)$ & $18(26)$ & \\
\hline Middle & $27(40)$ & $21(30)$ & \\
\hline Lower & $26(39)$ & $30(44)$ & \\
\hline Histological type, $n(\%)$ & & & 0.004 \\
\hline Intestinal & $38(57)$ & $22(32)$ & \\
\hline Diffuse & $29(43)$ & $47(68)$ & \\
\hline Tumor size (mm) & $43(7-110)$ & $71(13-180)$ & $<0.001$ \\
\hline Depth of invasion ${ }^{\mathrm{a}}, n(\%)$ & & & $<0.001$ \\
\hline $\mathrm{T} 1 / \mathrm{T} 2$ & $41(61)$ & $8(12)$ & \\
\hline $\mathrm{T} 3 / \mathrm{T} 4$ & $26(39)$ & $61(88)$ & \\
\hline Lymph node metastasis ${ }^{\mathrm{a}}, n(\%)$ & & & $<0.001$ \\
\hline N0/N1 & $49(73)$ & $23(33)$ & \\
\hline $\mathrm{N} 2 / \mathrm{N} 3$ & $18(27)$ & $46(67)$ & \\
\hline Distant metastasis $^{\mathrm{a}}, n(\%)$ & & & $<0.001$ \\
\hline M0 & $63(94)$ & $38(55)$ & \\
\hline M1 & $4(6)$ & $31(45)$ & \\
\hline
\end{tabular}

Continuous values are expressed as median (range). HBF, hepatic blood flow; High BF, greater than or equal to the median; BMI, body mass index; $\mathrm{Hb}$, hemoglobin; AST, aspartate aminotransferase; ALT, alanine aminotransferase; Cre, creatinine; SBP, systolic blood pressure. Low $\mathrm{HBF}$, less than the median $(<217 \mathrm{ml} / \mathrm{min} / 100 \mathrm{~g}$ tissue $)$. Include both pathological findings in operable patients and clinical findings in inoperable patients.

When patients were divided by the median value, the two groups differed significantly in body mass index (BMI), and blood hemoglobin $(\mathrm{Hb})$ level, whereas results were similar for liver, renal, and cardiac functional data. The high-HBF group contained more diffuse-type cancer (43\% vs. $68 \%$, $\mathrm{P}=0.004)$, larger tumors, (43 $\mathrm{mm}$ vs. $71, \mathrm{P}<0.001)$, and more advanced tumor-node stages (Table II). Mean HBFs by clinical disease stage were stage I: $183 \mathrm{ml} / \mathrm{min} / 100 \mathrm{~g}$ tissue (range, 133-323 ml/min/100 g tissue); stage II: $191 \mathrm{ml} / \mathrm{min} / 100 \mathrm{~g}$ tissue (range, $128-296 \mathrm{ml} / \mathrm{min} / 100 \mathrm{~g}$ tissue); stage III: $241 \mathrm{ml} / \mathrm{min} / 100 \mathrm{~g}$ tissue (range, 169-320 ml/min/100 g tissue); and stage IV: $263 \mathrm{ml} / \mathrm{min} / 100 \mathrm{~g}$ tissue (range, $176-430 \mathrm{ml} / \mathrm{min} / 100 \mathrm{~g}$ tissue). Patients with stage III/IV disease had significantly higher HBF than did those with stage I/II disease. We saw no difference between patients with stage I and II cancer $(\mathrm{P}=0.959)$ vs. those with stage III and IV cancer $(\mathrm{P}=0.088)$ (Fig. 2).

Postoperative change in $H B F$. Fourteen patients were excluded because of renal dysfunction by chemotherapy $(n=8)$, iodic allergic reaction $(n=4)$, and death within 6 months $(n=2)$ during postoperative follow-up. Comparing preoperative 


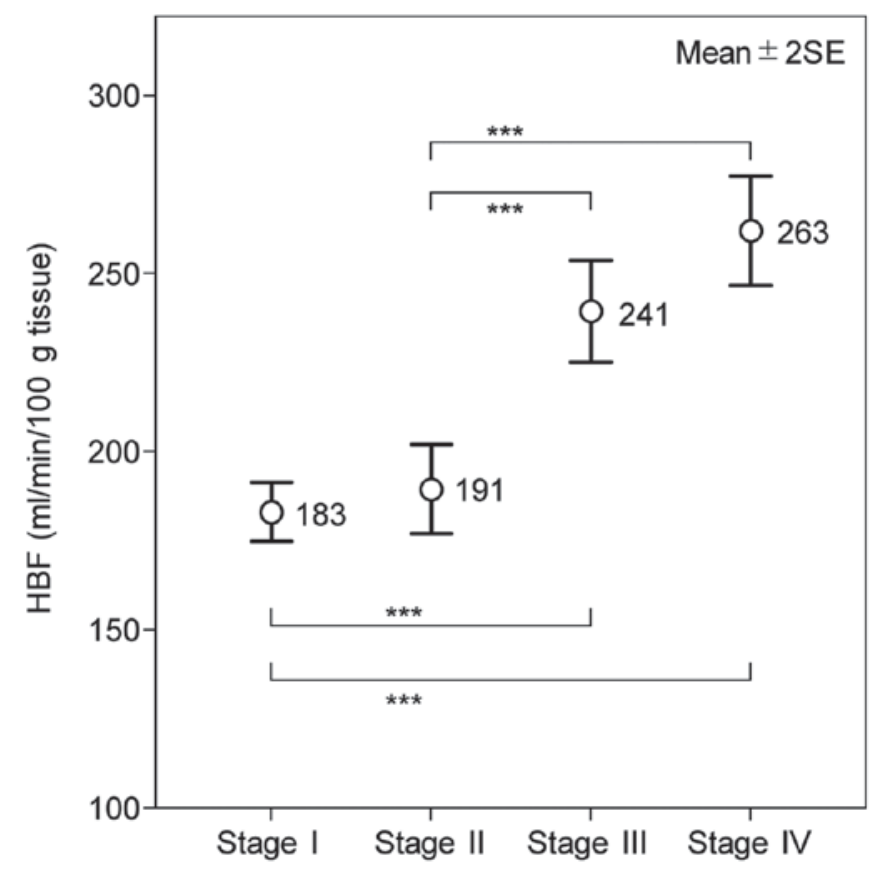

Figure 2. HBF at each clinical stage. ${ }^{* * *} \mathrm{P}<0.001 . \mathrm{HBF}$, hepatic blood flow; Stage, clinical stage; numerical number, mean value; SE, standard error.

HBF and results 6 months postoperatively, both were statistically similar in patients with pathological stage I/II disease ( $\mathrm{n}=56,183 \mathrm{ml} / \mathrm{min} / 100 \mathrm{~g}$ tissue vs. $188 \mathrm{ml} / \mathrm{min} / 100 \mathrm{~g}$ tissue, $\mathrm{P}=0.833$ ). Meanwhile postoperative HBF deceased significantly in patients with pathological stage III/IV disease $(n=33$, $232 \mathrm{ml} / \mathrm{min} / 100 \mathrm{~g}$ tissue vs. $207 \mathrm{ml} / \mathrm{min} / 100 \mathrm{~g}$ tissue, $\mathrm{P}=0.002$ ) (Fig. 3).
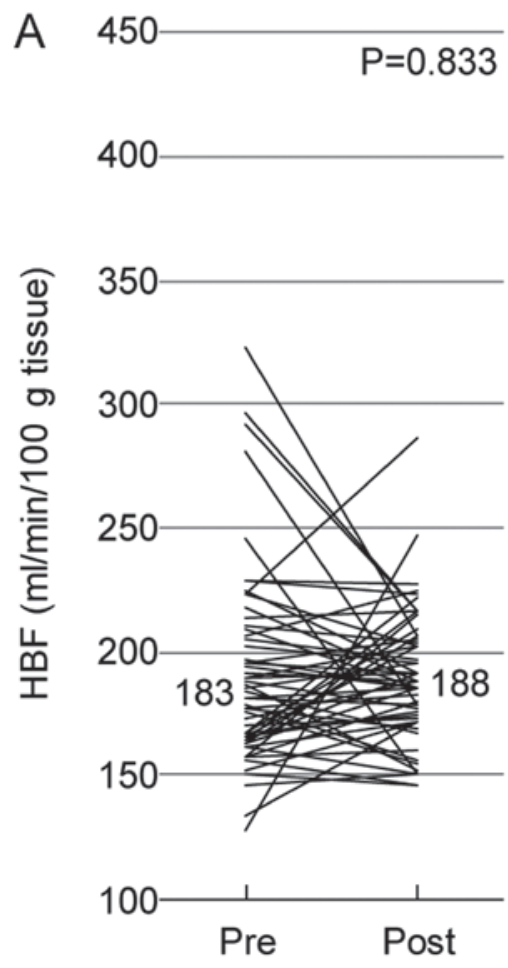

HBF and prognosis. Median $\mathrm{HBF}$ for operable vs. inoperable patients was $201 \mathrm{ml} / \mathrm{min} / 100 \mathrm{~g}$ tissue (range, $128-430 \mathrm{ml} / \mathrm{min} / 100 \mathrm{~g}$ tissue) vs. $258 \mathrm{ml} / \mathrm{min} / 100 \mathrm{~g}$ tissue (range, $188-394 \mathrm{ml} / \mathrm{min} / 100 \mathrm{~g}$ tissue). When both patient groups were compared by their respective median HBF values, the high-HBF group had a significantly worse prognosis $(\mathrm{P}=0.002$ and $\mathrm{P}=0.024)$, notably in the inoperable group, with $<1$-year survival (Fig. 4).

Postoperative HBF in patients with recurrent gastric cancer. The median postoperative follow-up duration was 740 days (range, 43-1923 days). Seventeen (17\%) patients had recurrent disease and a median $\mathrm{HBF}$ preoperative, 6 months' postoperatively, and recurrent of $212 \mathrm{ml} / \mathrm{min} / 100 \mathrm{~g}$ tissue (range, $128-430 \mathrm{ml} / \mathrm{min} / 100 \mathrm{~g}$ tissue), $191 \mathrm{ml} / \mathrm{min} / 100 \mathrm{~g}$ tissue (range, $141-375 \mathrm{ml} / \mathrm{min} / 100 \mathrm{~g}$ tissue), and $246 \mathrm{ml} / \mathrm{min} / 100 \mathrm{~g}$ tissue (range, $171-374 \mathrm{ml} / \mathrm{min} / 100 \mathrm{~g}$ tissue), respectively. $\mathrm{HBF}$ at disease recurrence was significantly higher compared with HBF 6 months postoperatively $(\mathrm{P}=0.002)$ and preoperatively $(\mathrm{P}=0.015)$. Two patients underwent repeat surgery. When recurrent patients were divided by the median $\mathrm{HBF}$ value at recurrence, the high-HBF group had a significantly worse postrecurrent prognosis ( $\mathrm{P}=0.019)$ (Fig. 5).

Prognostic significance of $H B F$. Clinical parameters assessed in Table II were evaluated by univariate analyses in all patients, then variables with significant difference were entered into the multivariate analysis. On behalf of cancer status, tumor depth and node metastasis were applied for analysis. As a result, $\mathrm{HBF}$ was an independent prognostic factor [hazard ratio $(\mathrm{HR}), 2.019 ; \mathrm{P}=0.048$ ] as were node
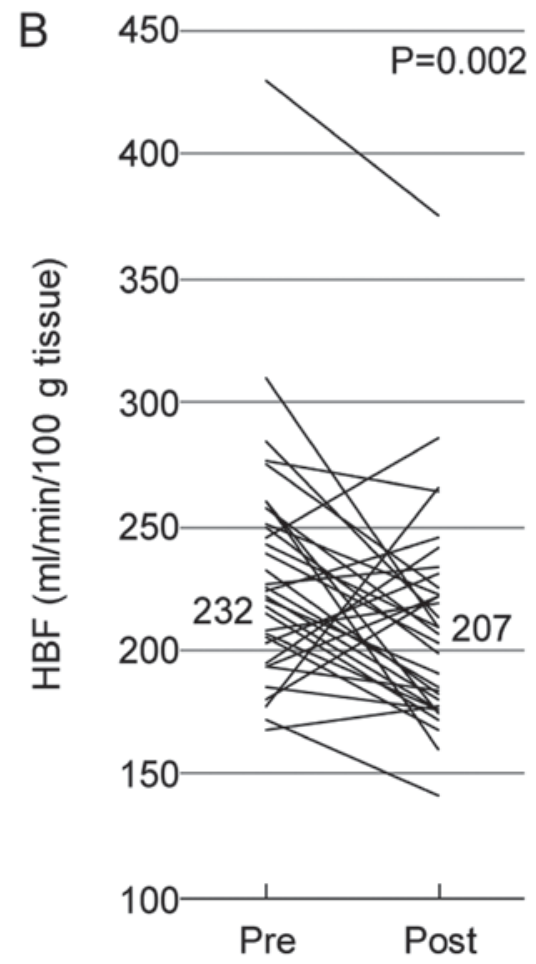

Figure 3. Postoperative change in HBF. HBF, hepatic blood flow; pre, preoperative; post, 6 months postoperatively; bold numerical number, median value. (A) Patients with pathological stage I/II disease $(n=56)$, (B) Patients with pathological stage III/IV disease $(n=33)$. 

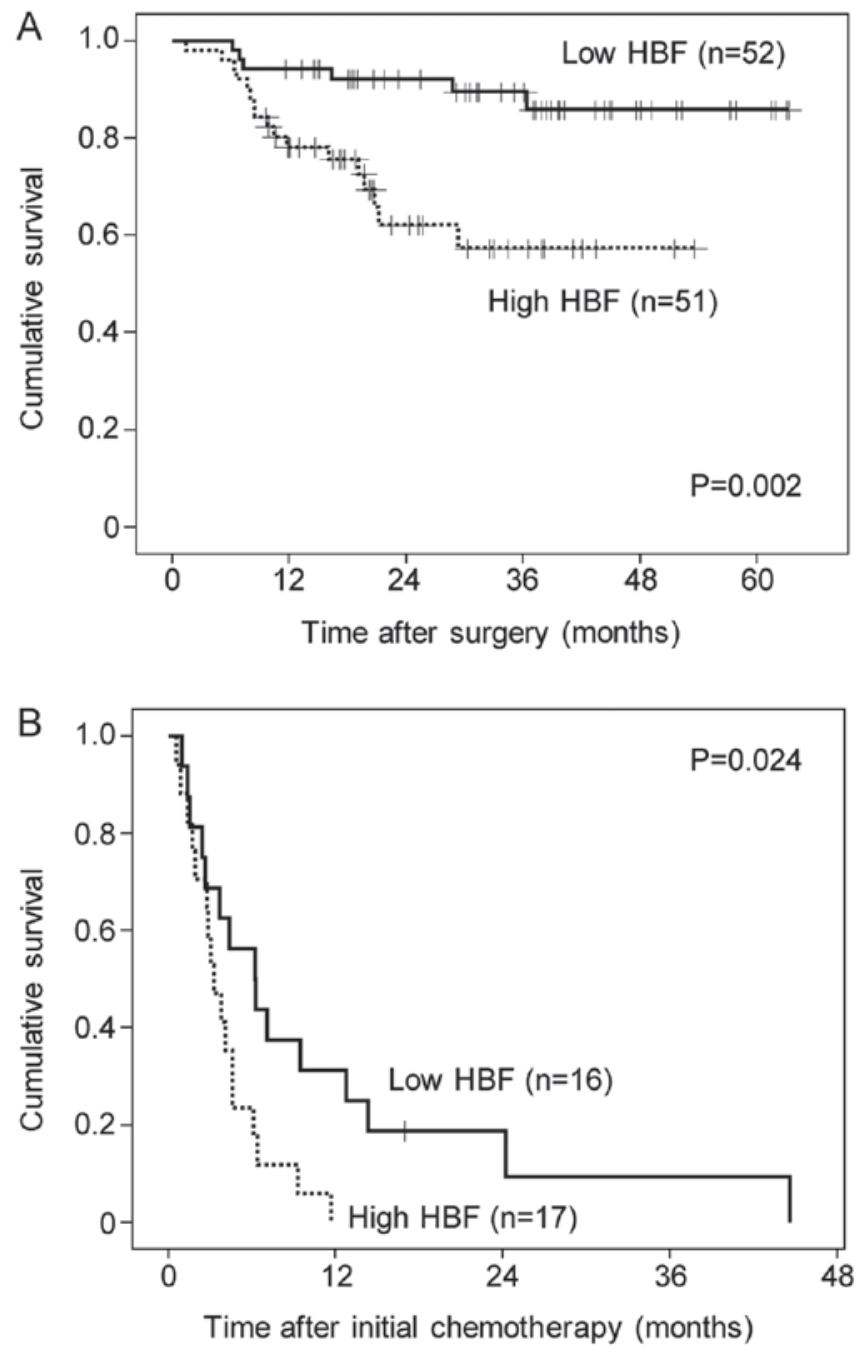

Figure 4. Overall survival curves of (A) operable and (B) inoperable patients. HBF, hepatic blood flow; Low HBF (solid line), less than the median HBF; High HBF (dashed line), greater than or equal to the median HBF.

metastasis (HR, 3.339; $\mathrm{P}=0.002)$ and histological type (HR, 2.259; $\mathrm{P}=0.014)$ (Table III).

HBF and serum proangiogenic cytokines. $\mathrm{HBF}$ was strongly correlated with VEGF $(\mathrm{R}=0.607, \mathrm{P}<0.001)$ and weakly correlated with $\mathrm{HGF}(\mathrm{R}=0.277, \mathrm{P}=0.083)$. No correlation was observed between HBF and either VEGFR-2 or angiopoietin-1 (Fig. 6).

Predictive factors for $H B F$. Clinical parameters assessed in Table II were evaluated by univariate analyses, then variables with significant difference were entered into the multifactorial analysis. As a result, BMI [odds ratio (OR), 4.754; $\mathrm{P}=0.001]$ and $\mathrm{Hb}(\mathrm{OR}, 2.576 ; \mathrm{P}=0.040)$ were independent predictive factors for $\mathrm{HBF}$ as was tumor depth (OR, 4.351; $\mathrm{P}=0.028)$ (Table IV).

\section{Discussion}

Previous applications of PCT for gastrointestinal cancer. Previous reports discussed the relationship between tumor blood flow assessed by PCT and malignant behaviors in tumors.
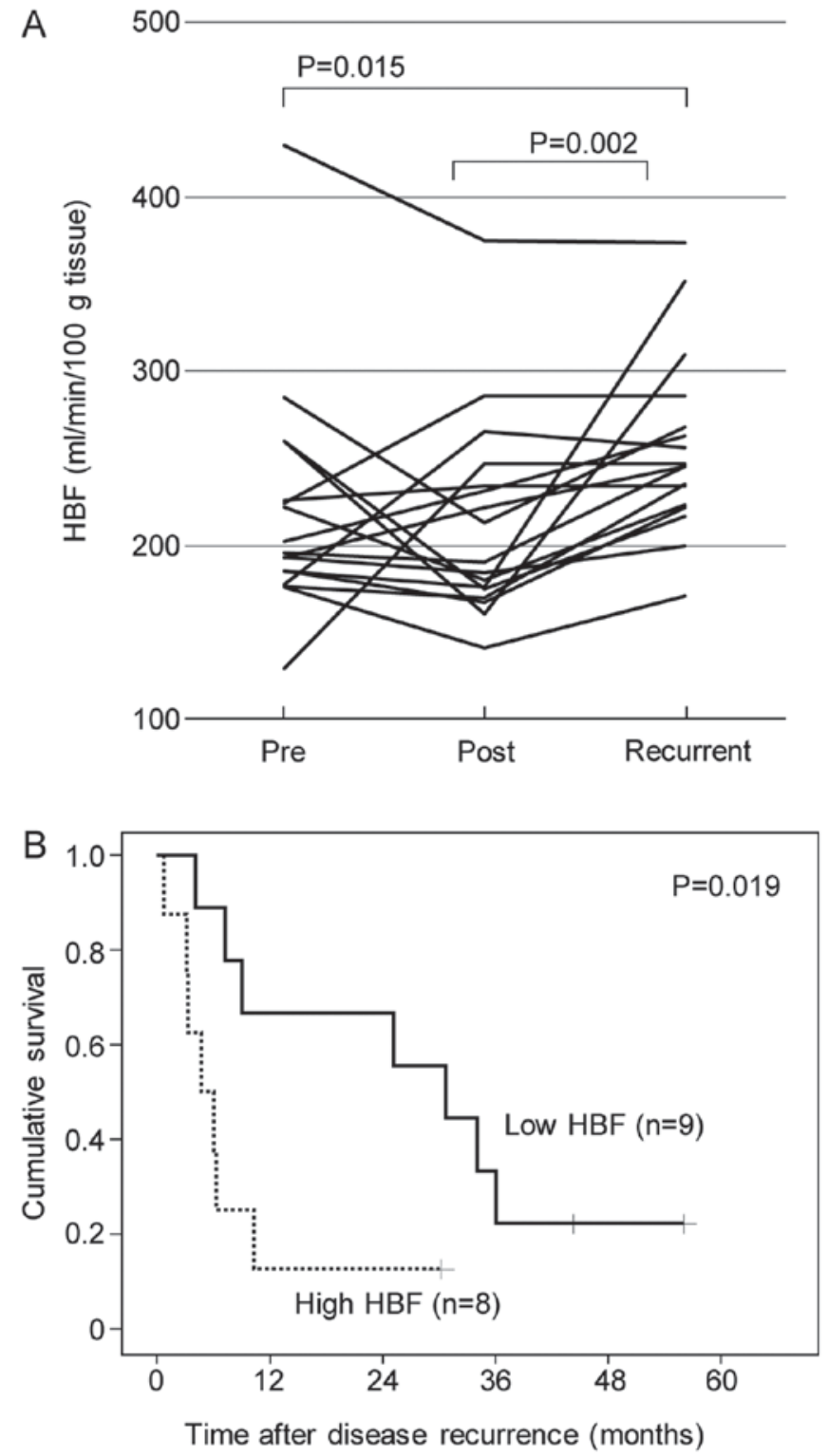

Figure 5. Postoperative HBF change and postrecurrent survival in patients with recurrent gastric cancer. (A) $\mathrm{HBF}$ at disease recurrence was significantly higher compared with HBF 6 months postoperatively $(246 \mathrm{ml} / \mathrm{min} / 100 \mathrm{~g}$ tissue vs. $191, \mathrm{P}=0.002)$ and preoperatively $(246 \mathrm{ml} / \mathrm{min} / 100 \mathrm{~g}$ tissue vs. 212 , $\mathrm{P}=0.015$ ). (B) When recurrent patients were divided according to the median $\mathrm{HBF}$ value at recurrence $(246 \mathrm{ml} / \mathrm{min} / 100 \mathrm{~g}$ tissue $)$, the high- $\mathrm{HBF}$ group had a significantly worse postrecurrent prognosis $(\mathrm{P}=0.019)$. HBF, hepatic blood flow.

Hayano et al demonstrated that tumors with low blood flow tended to be accompanied by synchronous metastatic lymph nodes or distant metastases and associated with poor patient survival in colorectal cancer (29). They concluded that tumor growth might lead to increase both tissue pressure and capillary compression and subsequently reduce tumor blood flow, as a result, hypoxic environment in tumor tissue might promote oncogenic mutations and finally more aggressive behaviors in tumor. Another paper reported that neoadjuvant therapy was more effective in patients with higher baseline tumor blood flow and patients with a positive treatment response showed decrease tumor blood flow in gastric cancer (30). Previous reports applied PCT for assessing tumor neovascularization and focused on predicting patient prognosis and response to 
Table III. Prognostic parameters by univariate and multivariate analysis.

\begin{tabular}{|c|c|c|c|c|c|c|c|c|}
\hline \multirow[b]{2}{*}{ Variables } & \multirow[b]{2}{*}{ Category } & \multirow[b]{2}{*}{$\mathrm{n}$} & \multicolumn{3}{|c|}{ Univariate analysis } & \multicolumn{3}{|c|}{ Multivariate analysis } \\
\hline & & & HR & $95 \%$ CI & $\mathrm{P}$-value & HR & $95 \% \mathrm{CI}$ & $\mathrm{P}$-value \\
\hline \multirow[t]{2}{*}{$\mathrm{BMI}\left(\mathrm{kg} / \mathrm{mm}^{2}\right)$} & $<21.5$ & 68 & 1.883 & $1.092-3.249$ & 0.023 & 1.108 & $0.605-2.031$ & 0.739 \\
\hline & $\geq 21.5$ & 68 & Reference & & & & & \\
\hline \multirow[t]{2}{*}{$\mathrm{Hb}(\mathrm{g} / \mathrm{dl})$} & $<12.6$ & 68 & 2.877 & $1.629-5.080$ & $<0.001$ & 1.361 & $0.754-2.458$ & 0.306 \\
\hline & $\geq 12.6$ & 68 & Reference & & & & & \\
\hline \multirow[t]{2}{*}{ Histological type } & Diffuse & 76 & 3.684 & $1.970-6.890$ & $<0.001$ & 2.259 & $1.183-4.314$ & 0.014 \\
\hline & Intestinal & 60 & Reference & & & & & \\
\hline \multirow[t]{2}{*}{ Tumor depth ${ }^{\mathrm{a}}$} & $\mathrm{T} 3 / \mathrm{T} 4$ & 87 & 9.089 & $3.589-23.017$ & $<0.001$ & 2.184 & $0.709-6.733$ & 0.174 \\
\hline & $\mathrm{T} 1 / \mathrm{T} 2$ & 49 & Reference & & & & & \\
\hline \multirow[t]{2}{*}{ Node metastasis ${ }^{\mathrm{a}}$} & $\mathrm{N} 2 / \mathrm{N} 3$ & 64 & 7.416 & $3.806-14.449$ & $<0.001$ & 3.339 & $1.587-7.281$ & 0.002 \\
\hline & N0/N1 & 72 & Reference & & & & & \\
\hline $\mathrm{HBF}$ & $\geq 217$ & 69 & 4.192 & $2.295-7.657$ & $<0.001$ & 2.019 & $1.006-4.051$ & 0.048 \\
\hline$(\mathrm{ml} / \mathrm{min} / 100 \mathrm{~g}$ tissue $)$ & $<217$ & 67 & Reference & & & & & \\
\hline
\end{tabular}

Continuous values are categorized by the median value. HR, hazard ratio; CI, confidence interval; BMI, body mass index; Hb, hemoglobin;

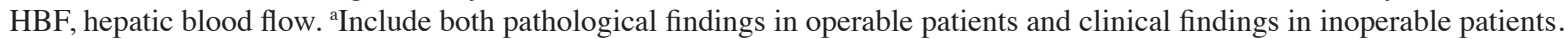
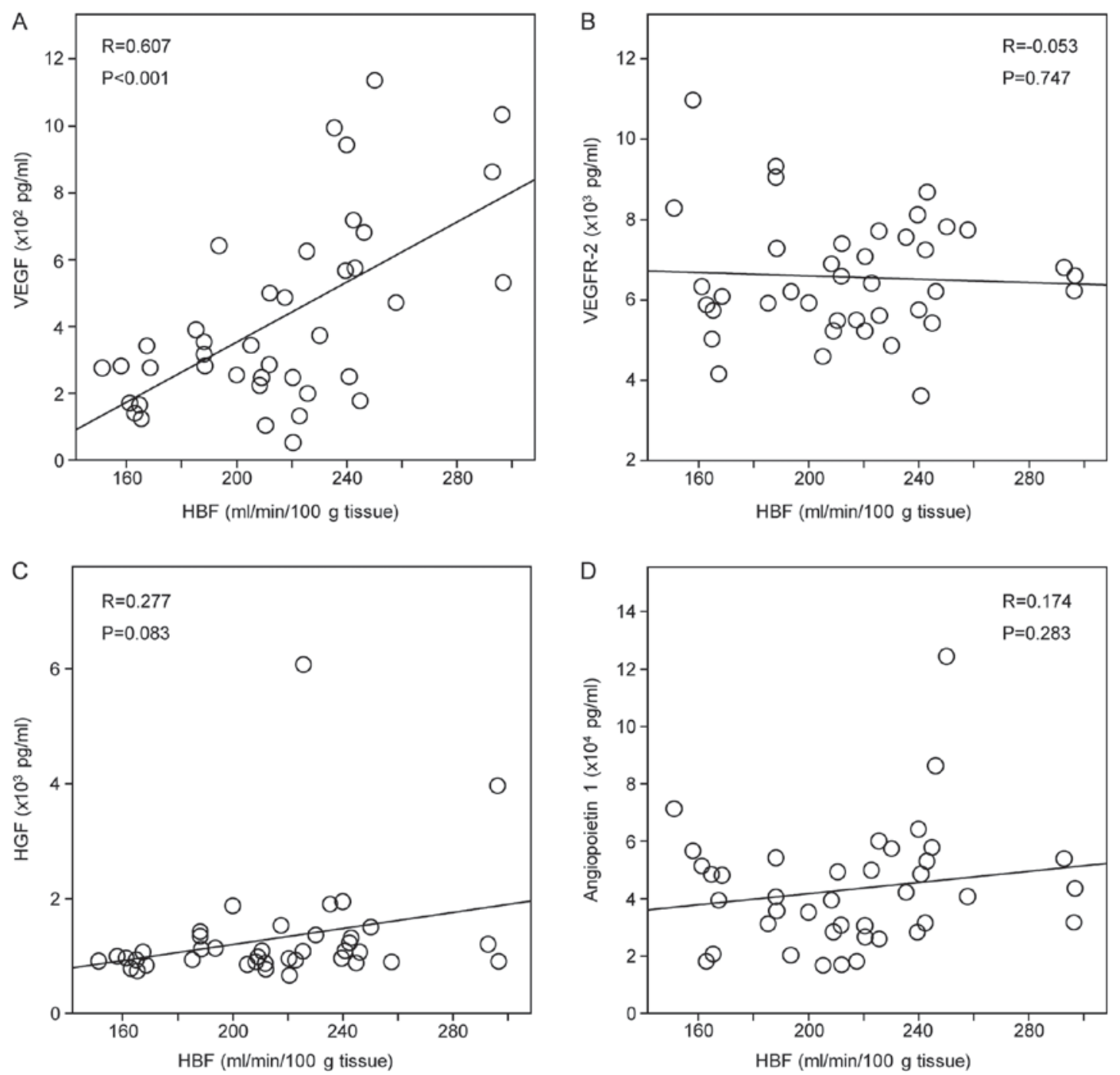

Figure 6. Correlation between HBF and serum proangiogenic cytokines. (A) HBF vs. VEGF, (B) HBF vs. VEGFR-2, (C) HBF vs. HGF, and (D) HBF vs. angiopoietin-1. HBF, hepatic blood flow; VEGF, vascular endothelial growth factor; VEGFR-2, VEGF receptor-2; HGF, hepatocyte growth factor. 
Table IV. Predictive factors for high hepatic blood flow by logistic regression analysis.

\begin{tabular}{|c|c|c|c|c|c|}
\hline Variables & Category & Regression coefficient & Odds ratio & $95 \% \mathrm{CI}$ & P-value \\
\hline Sex & Female & 0.224 & 1.251 & $0.396-3.949$ & 0.703 \\
\hline BMI $\left(\mathrm{kg} / \mathrm{mm}^{2}\right)$ & $<21.5$ & 1.520 & 4.754 & $1.815-11.523$ & 0.001 \\
\hline $\mathrm{Hb}(\mathrm{g} / \mathrm{dl})$ & $<12.6$ & 0.946 & 2.576 & $1.042-6.370$ & 0.040 \\
\hline Tumor size (mm) & $\geq 55$ & 0.148 & 1.159 & $0.398-3.373$ & 0.787 \\
\hline Histological type & Diffuse & 0.240 & 1.271 & $0.505-3.198$ & 0.661 \\
\hline Tumor depth ${ }^{\mathrm{a}}$ & $\mathrm{T} 3 / \mathrm{T} 4$ & 1.470 & 4.351 & $1.172-16.153$ & 0.028 \\
\hline Node metastasis ${ }^{\mathrm{a}}$ & $\mathrm{N} 2 / \mathrm{N} 3$ & 0.747 & 2.111 & $0.736-6.050$ & 0.164 \\
\hline
\end{tabular}

High hepatic blood flow, greater than or equal to the median ( $\geq 217 \mathrm{ml} / \mathrm{min} / 100 \mathrm{~g}$ tissue). Continuous values are categorized by the median

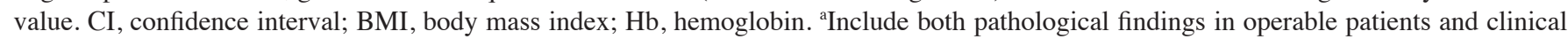
findings in inoperable patients.

chemotherapy or chemoradiotherapy (23-25). The application of present study is quite different from these previous reports. We assessed hepatic perfusion as a surrogate marker of tissue hemodynamic changes that might arise in the process of cancer progression.

HBF in patients with gastric cancer. Although previous reports demonstrated increased $\mathrm{HBF}$ in patients with synchronous liver metastasis with digestive cancer using scintigraphy and Doppler ultrasonography more than two decades ago $(31,32)$, HBF was not further discussed because of quantitative difficulties in its measurement. Since hepatic PCT was introduced into clinical practice, it has been recognized as a valid clinical method for early detection of obscure liver micrometastasis with incremental arterial flow by intrahepatic neovascularization (33-36). However, our concept differs from these studies because we evaluated cancer-associated systemic change using hepatic perfusion.

In this study, we demonstrated a relationship between $\mathrm{HBF}$ and gastric cancer progression. HBF increased in association with larger tumor size, increased depth of tumor invasion, lymph node metastasis, and stage. Postoperatively, HBF declined in resectable gastric cancer patients with advanced stage III/IV disease, and increased further in recurrent cases. The high-HBF group had a significantly worse prognosis in all patient groups: Operable, inoperable, and recurrent disease. Moreover, HBF was an independent prognostic imaging biomarker for gastric cancer in multivariate analysis, in our study. HBF might reflect the systemic change in tissue circulation developed by cancer progression. Patients with stage I cancer and with stage II had the same HBF; conversely, patients with stage III/IV cancer had significantly increased HBF levels. We presume that stage III disease may be systemic and require systemic therapies as well as locoregional treatment. This affirms the necessity of pre- and postoperative systemic chemotherapy for patients with stage III gastric cancer, currently a popular topic in gastric cancer treatment (37-39). To our knowledge, few studies have discussed cancer progression focusing on $\mathrm{HBF}(40)$.

Mechanism of increased $H B F$. Although we could not clarify the reason for the HBF increase in this study, we speculate two indirect mechanisms under cancer progression. The first is changes in a patient's general condition, and the second is the impact of circulating proangiogenic factors. The high-HBF group in our study had significantly lower BMI and lower $\mathrm{Hb}$ levels. BMI and $\mathrm{Hb}$ were also independent predictive factors for HBF by multifactorial analysis as were tumor depth. These results suggest that $\mathrm{HBF}$ may be influenced by $\mathrm{BMI}$ and $\mathrm{Hb}$ level as well as cancer status. We deem at the current moment that HBF may indirectly increase with lower abdominal pressure secondary to visceral fat loss and also may increase secondary to the intrahepatic vascular response to maintain hepatic perfusion with anemia. HBF might be a reflection of these changes in physical status associated with cancer progression.

Platelets isolated from cancer patients contain more VEGF than those from healthy controls $(41,42)$. Localized platelet aggregation may contribute to tumor progression by releasing a variety of vasoactive and proangiogenic substances in the tumor and may contribute to metastasis by facilitating adherence of disseminated tumor cells to capillary walls at distant sites $(43,44)$. We consider that these circulating vasoactive and proangiogenic substances in patients with advanced disease may affect hepatic perfusion. VEGF and, comparatively, HGF likely play roles in these vasoactive responses.

Clinical relevance of $H B F$. The clinical utility of serum tumor markers remains controversial. Although many tumor markers, such as carcinoembryonic antigen (CEA) and carbohydrate antigen 19-9 (CA19-9), have been studied in the monitoring of gastric cancer, none has sufficient sensitivity. The respective sensitivities of CEA and CA 19-9 for predicting gastric cancer recurrence are 31-66 and 16-55\%, which seems rather low (45). Postoperative HBF monitoring might predict cancer recurrence, and even in recurrent disease, aggressive or supportive treatment might be selected based on HBF in the future; however, further investigations are needed in this respect.

Limitations. Since HBF is evaluated by dynamic CT imaging during a nearly 1-min breath hold, excessive respiratory movement prevents accurate analysis. Moreover, we were not able to clarify the normal range for HBF in this preliminary study. 
Further study is warranted to identify the relationship between $\mathrm{HBF}$ and cancer progression and recurrence including hepatic histological evaluations in larger samples.

In conclusion, $\mathrm{HBF}$ increases in association with gastric cancer progression. Patients with advanced gastric cancer and recurrent disease had significantly increased HBF. HBF may be an easily assessed and independent imaging biomarker for predicting patient survival.

\section{Acknowledgements}

The authors would like to thank Professor Munemasa Ryu (Chairman of Medical Welfare Network Chiba, Chiba, Japan) for providing advice during the study.

\section{Funding}

This study was supported by nonprofit organization grants from the Cancer Research Funds for Patients and Family, Medical Welfare Network Chiba (MWNC-NPO) (Chiba, Japan).

\section{Availability of data and materials}

The datasets analyzed during the current study are available from the corresponding author on reasonable request.

\section{Authors' contributions}

KS, TF and AS concepted and designed of study. MM, CK and $\mathrm{KN}$ acquired imaging data. $\mathrm{KS}, \mathrm{KH}$ and $\mathrm{SN}$ analyzed and interpreted the patient data. MM performed the histological evaluation. HS and KK contributed in acquiring clinicopathological data. All authors read and approved the final manuscript.

\section{Ethics approval and consent to participate}

This study was approved by the institutional review board (IRB) of the Medical Ethical Committee of Teikyo University (Ichihara, Japan), and written informed consent was obtained from all patients (IRB number, 12-179; UMIN000019970 and IRB number, 15-007; UMIN000019973).

\section{Patient consent for publication}

Written informed consent was obtained from the patient for publication of this research.

\section{Competing interests}

The authors declare that they have no competing interests.

\section{References}

1. Globocan 2012: Estimated cancer incidence, mortality and prevalence worldwide in 2012 (Internet). Lyon, International Agency for Research on Cancer, 2013. http://globocan.iarc.fr.

2. Sakuramoto S, Sasako M, Yamaguchi T, Kinoshita T, Fujii M, Nashimoto A, Furukawa H, Nakajima T, Ohashi Y, Imamura $\mathrm{H}$, et al: Adjuvant chemotherapy for gastric cancer with S-1, an oral fluoropyrimidine. N Engl J Med 357: 1810-1820, 2007.
3. GASTRIC (Global Advanced/Adjuvant Stomach Tumor Research International Collaboration) Group, Oba K, Paoletti X, Bang YJ, Bleiberg H, Burzykowski T, Fuse N, Michiels S, Morita S, Ohashi Y, et al: Role of chemotherapy for advanced/recurrent gastric cancer: An individual-patient-data meta-analysis. Eur J Cancer 49: 1565-1577, 2013.

4. Terashima M, Ichikawa W, Ochiai A, Kitada K, Kurahashi I, Sakuramoto S, Katai H, Sano T, Imamura H and Sasako M; ACTS-GC Group: TOP2A, GGH, and PECAM1 are associated with hematogenous, lymph node, and peritoneal recurrence in stage II/III gastric cancer patients enrolled in the ACTS-GC study. Oncotarget 8: 57574-57582, 2017.

5. Heber D, Byerly LO and Chlebowski RT: Metabolic abnormalities in the cancer patient. Cancer 55 (Suppl 1): S225-S229, 1985.

6. Stockert E, Jäger E, Chen YT, Scanlan MJ, Gout I, Karbach J, Arand M, Knuth A and Old LJ: A survey of the humoral immune response of cancer patients to a panel of human tumor antigens. J Exp Med 187: 1349-1354, 1998.

7. Dranoff G: Cytokines in cancer pathogenesis and cancer therapy. Nat Rev Cancer 4: 11-22, 2004

8. Hanahan D and Folkman J: Patterns and emerging mechanisms of the angiogenic switch during tumorigenesis. Cell 86: 353-364, 1996.

9. Holmgren L, O'Reilly MS and Folkman J: Dormancy of micrometastases: Balanced proliferation and apoptosis in the presence of angiogenesis suppression. Nat Med 1: 149-153, 1995.

10. Ferrara N, Gerber HP and LeCouter J: The biology of VEGF and its receptors. Nat Med 9: 669-676, 2003.

11. Maeda K, Chung YS, Ogawa Y, Takatsuka S, Kang SM, Ogawa M, Sawada T and Sowa M: Prognostic value of vascular endothelial growth factor expression in gastric carcinoma. Cancer 77: 858-863, 1996.

12. Park DJ, Thomas NJ, Yoon C and Yoon SS: Vascular endothelial growth factor an inhibition in gastric cancer. Gastric Cancer 18: 33-42, 2015.

13. Lee EY, Cibull ML, Strodel WE and Haley JV: Expression of HER-2/neu oncoprotein and epidermal growth factor receptor and prognosis in gastric carcinoma. Arch Pathol Lab Med 118: 235-239, 1994.

14. Lordick F: Targeting the HGF/MET pathway in gastric cancer. Lancet Oncol 15: 914-916, 2014.

15. Wang L, Chang Y, Xu J and Zhang Q: Predictive significance of serum level of vascular endothelial growth factor in gastric cancer patients. Biomed Res Int 2016: 8103019, 2016.

16. Karayiannakis AJ, Syrigos KN, Polychronidis A, Zbar A, Kouraklis G, Simopoulos C and Karatzas G: Circulating VEGF levels in the serum of gastric cancer patients: Correlation with pathological variables, patient survival, and tumor surgery. Ann Surg 236: 37-42, 2002.

17. Park DJ, Seo AN, Yoon C, Ku GY, Coit DG, Strong VE, Suh YS, Lee HS, Yang HK, Kim HH and Yoon SS: Serum VEGF-A and tumor vessel VEGFR-2 levels predict survival in caucasian but not asian patients undergoing resection for gastric adenocarcinoma. Ann Surg Oncol 22 (Suppl 3): S1508-S15015, 2015.

18. Jüttner S, Wissmann C, Jöns T, Vieth M, Hertel J, Gretschel S, Schlag PM, Kemmner W and Höcker M: Vascular endothelial growth factor-D and its receptor VEGFR-3: Two novel independent prognostic markers in gastric adenocarcinoma. J Clin Oncol 24: 228-240, 2006.

19. Axel L: Cerebral blood flow determination by rapid-sequence computed tomography: Theoretical analysis. Radiology 137: 679-686, 1980

20. Miles KA, Hayball M and Dixon AK: Colour perfusion imaging: A new application of computed tomography. Lancet 337: 643-645, 1991

21. Ronot M, Asselah T, Paradis V, Michoux N, Dorvillius M, Baron G, Marcellin P, Van Beers BE and Vilgrain V: Liver fibrosis in chronic hepatitis $C$ virus infection: Differentiating minimal from intermediate fibrosis with perfusion CT. Radiology 256: 135-142, 2010.

22. Hashimoto K, Murakami T, Dono K, Hori M, Kim T, Kudo M, Marubashi S, Miyamoto A, Takeda Y, Nagano $\mathrm{H}$, et al: Assessment of the severity of liver disease and fibrotic change: The usefulness of hepatic CT perfusion imaging. Oncol Rep 16: 677-683, 2006

23. Hayano K, Okazumi S, Shuto K, Matsubara H, Shimada H, Nabeya Y, Kazama T, Yanagawa N and Ochiai T: Perfusion CT can predict the response to chemoradiation therapy and survival in esophageal squamous cell carcinoma: Initial clinical results. Oncol Rep 18: 901-908, 2007. 
24. Satoh A, Shuto K, Okazumi S, Ohira G, Natsume T, Hayano K, Narushima K, Saito H, Ohta T, Nabeya Y, et al: Role of perfusion $\mathrm{CT}$ in assessing tumor blood flow and malignancy level of gastric cancer. Dig Surg 27: 253-260, 2010.

25. García-Figueiras R, Goh VJ, Padhani AR, Baleato-González S, Garrido M, León L and Gómez-Caamaño A: CT perfusion in oncologic imaging: A useful tool? AJR Am J Roentgenol 200: 8-19, 2013.

26. James DB, Mary KG and Christian W (eds): TNM Classification of Malignant Tumours. 8th edition. Wiley-Liss, New York, 2017.

27. Japanese Gastric Cancer Association: Japanese Classification of Gastric Carcinoma. 15th edition. Kanehara Publisher, Tokyo, pp27-23, 2017.

28. Meier P and Zierler KL: On the theory of the indicator-dilution method for measurement of blood flow and volume. J Appl Physiol 6: 731-744, 1954.

29. Hayano K, Fujishiro T, Sahani DV, Satoh A, Aoyagi T, Ohira G, Tochigi T, Matsubara $\mathrm{H}$ and Shuto K: Computed tomography perfusion imaging as a potential imaging biomarker of colorectal cancer. World J Gastroenterol 20: 17345-17351, 2014.

30. Kruk-Bachonko J, Krupski W, Czechowski M, Kurys-Denis E, Mądro P, Sierocińska-Sawa J, Dąbrowski A, Wallner G and Skoczylas T: Perfusion CT-A novel quantitative and qualitative imaging biomarker in gastric cancer. Eur J Radiol 95: 399-408, 2017.

31. Leveson SH, Wiggins PA, Giles GR, Parkin A and Robinson PJ: Deranged liver blood flow patterns in the detection of liver metastases. Br J Surg 72: 128-130, 1985.

32. Leen E, Goldberg JA, Robertson J, Sutherland GR and McArdle CS: The use of duplex sonography in the detection of colorectal hepatic metastases. Br J Cancer 63: 323-325, 1991.

33. Miles KA, Hayball MP and Dixon AK: Functional images of hepatic perfusion obtained with dynamic CT. Radiology 188 : 405-411, 1993.

34. Nott DM, Grime SJ, Yates J, Day DW, Baxter JN, Jenkins SA and Cooke TG: Changes in the hepatic perfusion index during the development of experimental hepatic tumours. Br J Surg 76: 259-263, 1989.

35. Leggett DA, Kelley BB, Bunce IH and Miles KA: Colorectal cancer: Diagnostic potential of CT measurements of hepatic perfusion and implications for contrast enhancement protocols. Radiology 205: 716-720, 1997.

36. Cuenod CA, Leconte I, Siauve N, Resten A, Dromain C, Poulet B, Frouin F, Clément $\mathrm{O}$ and Frija G: Early changes in liver perfusion caused by occult metastases in rats: Detection with quantitative CT. Radiology 218: 556-561, 2001.
37. Cunningham D, Allum WH, Stenning SP, Thompson JN Van de Velde CJ, Nicolson M, Scarffe JH, Lofts FJ, Falk SJ, Iveson TJ, et al: Perioperative chemotherapy versus surgery alone for resectable gastroesophageal cancer. N Engl J Med 355: 11-20, 2006.

38. Yoshikawa T, Sasako M, Yamamoto S, Sano T, Imamura H, Fujitani K, Oshita H, Ito S, Kawashima Y and Fukushima N: Phase II study of neoadjuvant chemotherapy and extended surgery for locally advanced gastric cancer. Br J Surg 96: 1015-1012, 2009.

39. Smalley SR, Benedetti JK, Haller DG, Hundahl SA, Estes NC, Ajani JA, Gunderson LL, Goldman B, Martenson JA, Jessup JM, et al: Updated analysis of SWOG-directed intergroup study 0116: A phase III trial of adjuvant radiochemotherapy versus observation after curative gastric cancer resection. J Clin Oncol 30: 2327-2333, 2012.

40. Fujishiro T, Shuto K, Hayano K, Satoh A, Kono T, Ohira G, Tohma T, Gunji H, Narushima K, Tochigi T, et al: Preoperative hepatic CT perfusion as an early predictor for the recurrence of esophageal squamous cell carcinoma: Initial clinical results. Oncol Rep 31: 1083-1088, 2014.

41. Salven P, Orpana A and Joensuu H: Leukocytes and platelets of patients with cancer contain high levels of vascular endothelial growth factor. Clin Cancer Res 5: 487-491, 1999.

42. Lee JK, Hong YJ, Han CJ, Hwang DY and Hong SI: Clinical usefulness of serum and plasma vascular endothelial growth factor in cancer patients: Which is the optimal specimen? Int J Oncol 17: 149-152, 2000.

43. Browder T, Folkman J and Pirie-Shepherd S: The hemostatic system as a regulator of angiogenesis. J Biol Chem 275: 1521-1524, 2000.

44. Hejna M, Raderer M and Zielinski CC: Inhibition of metastases by anticoagulants. J Natl Cancer Inst 91: 22-36, 1999.

45. Takahashi Y, Takeuchi T, Sakamoto J, Touge T, Mai M, Ohkura H, Kodaira S, Okajima K and Nakazato H; Tumor Marker Committee: The usefulness of CEA and/or CA19-9 in monitoring for recurrence in gastric cancer patients: A prospective clinical study. Gastric Cancer 6: 142-145, 2003.

This work is licensed under a Creative Commons Attribution-NonCommercial-NoDerivatives 4.0 International (CC BY-NC-ND 4.0) License. 